\title{
Ethnobotanical study of curative plants used by traditional healers to treat rhinitis in the Limpopo Province, South Africa
}

\author{
Sebua Silas Semenya ${ }^{1,2^{*}}$, Alfred Maroyi ${ }^{2}$
}

1. Technology Transfer office, Research Administration and Development Department, University of Limpopo, Private Bag X1106, Sovenga 0727, South Africa.

2. Medicinal Plants and Economic Development (MPED) Research Centre, Department of Botany, University of Fort Hare, Private Bag X1314, Alice 5700, South Africa.

\begin{abstract}
Background: Studies focusing on plants used medicinally to heal and manage rhinitis are very scarce in Africa and elsewhere. Objectives: To document plants used by traditional healers (THs) in the treatment and management of rhinitis and related symptoms in the Limpopo Province of South Africa.

Methods: Data was gathered using a semi structured interviews with 105 THs in the Limpopo Province, supplemented by field expeditions for plant specimen collection and observation.

Results: 63 plant species from 59 genera distributed across 40 botanical families, mainly the Asteraceae $(7$ spp.) and Euphorbiaceae (6 spp.) were therapeutically used by THs. Overall, 77 herbal recipes $(87 \%=$ mono and $12.9 \%=$ poly), mainly prepared from roots $(55.5 \%)$ and leaves $(19 \%)$ were recorded. Most of these recipes were processed via pounding (59.7\%) and boiling (20.7\%). Oral (44.1\%) and nasal (33.7\%) was the preferred modes of dispensing remedies by THs. Artemisia afra, Clerodendrum ternatum, Cryptocarya transvaalensis, Enicostema axillare, Kalanchoe brachyloba, Lasiosiphon caffer, Lippia javanica, Schkubria pinnata, Securidaca longepedunculata, Spirostachys africana, Stylochaeton natalensis and Zanthoxylum capense were the most widely used and preferred species for treatment of rhinitis and/or related symptom by all questioned THs.

Conclusion: This study is the first to document plants used traditionally to treat rhinitis in Africa. Therefore, it provides a baseline data on the plants used to heal rhinitis in the Limpopo Province. Finding of this study can be used as apedestal for further investigation in to effective plant-based anti-rhinitis drugs.
\end{abstract}

Keywords: Bapedi, Limpopo, traditional healers, rhinitis.

DOI: https://dx.doi.org/10.4314/ahs.v18i4.29

Cite as: Semenya SS, Maroyi A. Ethnobotanical study of curative plants used by traditional healers to treat rbinitis in the Limpopo Province, South Africa. Afri Health Sci. 2018;18(4): 1076-1087. https:/ / dx.doi.org/10.4314/ahs.v18i4.29

\section{Introduction}

Rhinitis is a respiratory affliction that is characterised by inflammation of the nasal mucosa ${ }^{1}$. This complaint can either be allergic and non-allergic. Overall, rhinitis is a significant cause of both the widespread morbidity and medical treatment costs in many countries worldwide. It is estimated that over 400 million people inhabiting both developed and developing countries are diagnosed with

\section{Corresponding author:}

Sebua Silas Semenya, Technology Transfer office, Research

Administration and Development Department, University of Limpopo,

Private Bag X1106, Sovenga 0727, South Africa.

Email: sebuasemenya@gmail.com this condition ${ }^{2}$. For instance, up to $40 \%$ of children, and approximately $20 \%$ of the adult population in the United States of America were previously reported to suffer from rhinitis ${ }^{3}$. The latter occurrence is lower compared to $29.8 \%$ noted by Morais-Almeida et al. ${ }^{4}$ amongst the adult subjects residing in Portugal. Eriksson ${ }^{5}$ reported 26.9\% prevalence of rhinitis amid the West Swedenees population.

Incidence of rhinitis is also common in Africa, and here it varies according to the geographical location as well as age group. In Nigeria the prevalence of $29.6 \%$ amongst adults aged between 18 and 45 years was previously registered ${ }^{6}$. The occurrence of rhinitis in this age brackets was $10.4 \%$ in Cameroon ${ }^{7}$. This respiratory infection affected at least $15.3 \%$ of Egyptian population aged $11-15$ year

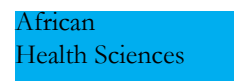

1076
(C) 2018 Semenya et al. Licensee African Health Sciences. This is an Open Access article distributed under the terms of the Creative commons Attribution License (https://creativecommons.org/licenses/BY/4.0), which permits unrestricted use, distribution, and reproduction in any medium, provided the original work is properly cited.

African Health Sciences Vol 18 Issue 4, December, 2018 
olds $^{8}$. In South Africa, there are extremely few epidemiological studies that addressed the prevalent of rhinitis. However, available study; Zar et al. ${ }^{9}$, was conducted in two phases carried out seven years apart, amongst the 13-14 year olds adolescents in randomly selected South African schools. The outcomes of this surveys indicated that the incidence of rhinitis amongst these subjects had increased significantly between two time points. For instance, in phase I of the investigation, conducted in 1995, the prevalence was $30.4 \%$, and by phase II in 2003 , the occurrence had gone up to $38.5 \%$. The possible reasons for this drastic increase in the incidence of rhinitis could be attributed to the increasing prevalence of contributing factors.

Although sometimes rhinitis is mistakenly viewed as a trivial disease, its symptoms may significantly affect a patient's quality of life $^{10}$. Different disorders that are associated to this respiratory infection include bronchial asthma, sinusitis, otitis media, nasosinusal polyposis, respiratory infections and alterations in dental occlusion ${ }^{11}$. In addition, conditions such as headache, cognitive impairment, nasal obstruction, nasal itching, sneezing and watery rhinorrhoea are also common in rhinitis suffer ${ }^{12}$. Therefore, appropriate management of this respiratory affliction may be an important component in effective management of all the above stated coexisting or complicating respiratory ailments.

According to DeGuzman et al. ${ }^{13}$, management approaches of rhinitis by western health care professionals in Africa and elsewhere include educating patients about the various strategies to avoid risk factors such as animals and insect allergens, house dust mites, molds and pollens, amongst other factors. Therapeutic options or pharmacological agents such as anti-H1 antihistamines, intranasal corticosteroids, topical anticholinergic, topical or oral vasoconstrictors and sodium cromoglycate are also prescribed by these professionals as part of rhinitis treatment and management ${ }^{14}$. Despite the evidence indicating prevalence of rhinitis and its management approaches employed by western health professionals; little is still known about the herbal medications implicated by the aboriginal health care providers as treatment of this affliction. However, few attempts to address this gap in knowledge have been made in countries such as Turkey ${ }^{15}$ and India ${ }^{16}$. In Africa as a continent wherein over $80 \%$ of the population relies on traditional medicines and healers' health care services either as the only option or the preferred option ${ }^{17}$, ethnobotanical studies focusing on the treatment of rhinitis are lacking. The aim of the present study is therefore, to investigate the therapeutic plants implicated by traditional healers in the Limpopo Province against rhinitis.

\section{Methods}

\section{Study area and population}

The area selected for survey was the municipalities of Capricorn, Sekhukhune and Waterberg districts, in the Limpopo Province, South Africa (Figure 1). Five villages from each municipality were selected as study sites. Generally, most of these settlements are poorly developed and lack basic infrastructures such as sanitations, roads and healthcare facilities.

The populace of the sampled districts comprised of different ethnic groups, with Bapedi who speak Sepedi language being the largest group, comprising about $50 \%$ of the entire population ${ }^{18}$.

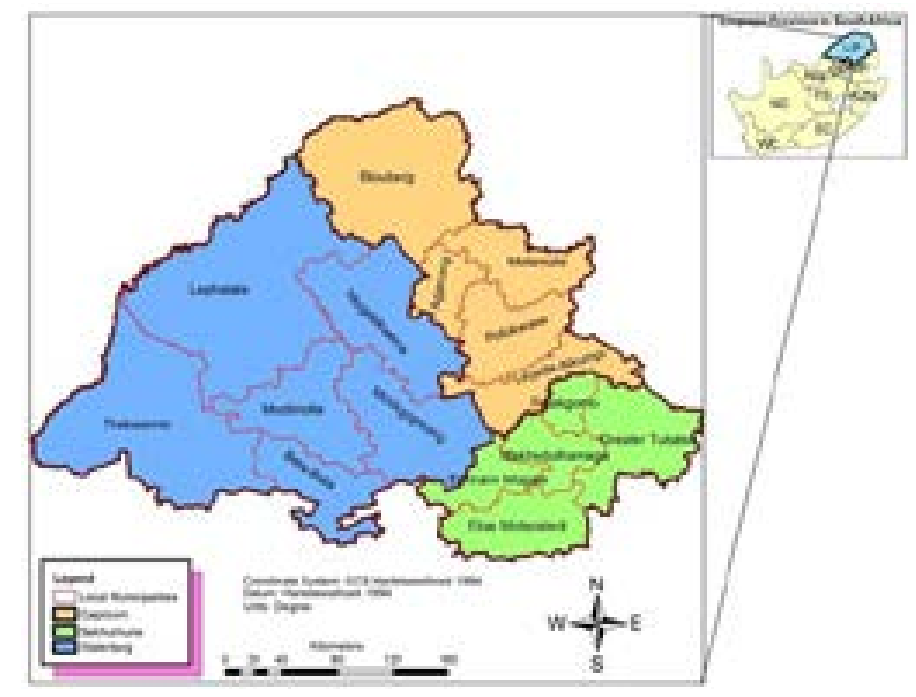

Figure 1: Map of Limpopo Province indicating the studied areas (districts and municipalities). 


\section{Ethnobotanical survey and data collection}

The ethical approval to conduct the present study was obtained from the Ethical Review Committee of the University of Fort Hare (Ref: MAR001SSEMO1). Prior to the field survey, permission to conduct an investigation was sought from the local tribal leaders of each sampled village, and subsequently THs were requested to take part in this study. Both the local community leaders and all the THs were informed about the purpose of the survey. Traditional healers who agreed to participate in the study were asked to sign a consent form before being questioned.

Data was collected from May 2017 to October 2017 using semi structure questionnaires during face-to-face interview with 105 THs who were conveniently chosen (i.e., with the help of local leaders and THs). The questionnaire was designed to captured information on the local name of used medicinal plants by THs to treat rhinitis, used plant part/s, mode of herbal preparation, administration and dosage prescription. Interviews were conducted by researchers, independently for each traditional healer in Sepedi language.

The data obtained from questionnaire guides was supplemented by independent field walk with each traditional healer for the practical identification of used medicinal plants and direct observations on their characteristics. During field trips plants were initially pointed-out by THs via vernacular names and subsequently researchers collected the voucher specimens. The collected plant samples were prepared (i.e., numbered, pressed and dried) and deposited at the Larry Leach Herbarium (University of Limpopo) for scientific identification by a trained taxonomist.

\section{Data analysis}

Micro Soft Excel and Statistical Package for the Social Sciences (SPSS)

Data from ethnobotanical surveys was analysed using Microsoft Excel 2000 and SPSS programmes by means of descriptive statistics. These programmes were used to identify proportions of plant families, habits, used species and their parts, condition of medicinal plant part/s use (fresh/dried), mode of remedies preparations and administration, amongst the other information.

\section{Fidelity level (FL)}

The FL was employed to quantify the percentages of THs claiming the use of a certain plant for rhinitis and/ related symptom/s. This was determined following the method of Al-Quran ${ }^{19}$ :

$$
\text { FL }(\%)=\frac{N P}{N} \times 100
$$

Where $\mathrm{Np}$ was the number of THs who independently used a particular plant species to treat rhinitis and/or related symptom, and $\mathrm{N}$ was the total number of THs who reported the plant as a medicine to treat any given disease (rhinitis or related symptom/s). The FL index shows the degree of homogeneity on the informant's knowledge regarding the medical application of a particular medicinal plant species against a specific ailment ${ }^{19}$, therefore it reveals the most utilised and avoided species.

\section{Use value (UV)}

Use value provides a good measure to estimate all the possible uses of an individual plant species. An individual plant-disease-use combination mentioned by Bapedi THs for rhinitis and related symptoms was evaluated using the formula outlined below ${ }^{20}$ :

$$
\mathrm{UV}=\Sigma \frac{U}{N}
$$

Where $U$ was the number of citations per species, and was $\mathrm{N}$ the total number of questioned THs. Generally, UV for a plant is high when there are corresponding broad therapeutic applications coupled with a relatively high use mentioned (UM) by participants ${ }^{20}$.

\section{Results \\ Diversity of used plant species}

In the present study a total of 63 plant species (55 indigenous and 8 exotics) from 59 genera distributed across 40 botanical families, mainly the Asteraceae (7 spp.), Euphorbiaceae (6 spp.) and Malvaceae (4 spp.) were used by 105 Bapedi THs to treat rhinitis and various symptoms they perceived as related to this respiratory infection (Table 1). Amongst these plant species, only $19 \%(n=12)$ comprising of Artemisia afra, Clerodendrum ternatum, Cryptocarya transvaalensis, Enicostema axillare, Kalanchoe brachyloba, Lasiosiphon caffer, Lippia javanica, Schkubria pinnata, Securidaca longepedunculata, Spirostachys africana, Stylochaeton natalensis and Zanthoxylum capense were most widely distributed and used by all THs $(n=105)$ who treated rhinitis across the studied districts and municipalities.

African Health Sciences Vol 18 Issue 4, December, 2018 
Table 1: Medicinal plants used by Bapedi traditional healers to treat rhinitis and related symptoms in the Limpopo Province, South Africa

\begin{tabular}{|c|c|c|c|c|c|c|c|c|c|c|c|}
\hline \multirow[t]{2}{*}{ Botanical family } & \multirow[t]{2}{*}{ Species name } & \multirow[t]{2}{*}{ Vernacular name } & \multirow[t]{2}{*}{ Habit } & \multirow[t]{2}{*}{$\begin{array}{l}\text { Used } \\
\text { plant } \\
\text { parts }\end{array}$} & \multirow[t]{2}{*}{$\begin{array}{l}\text { State } \\
\text { of use }\end{array}$} & \multirow[t]{2}{*}{$\begin{array}{l}\text { Methods of herbal } \\
\text { preparation and } \\
\text { administration }\end{array}$} & \multirow[t]{2}{*}{$\begin{array}{l}\text { Aliment } \\
\text { / } \\
\text { streated }\end{array}$} & \multicolumn{2}{|c|}{$\begin{array}{l}\text { Frequency } \\
\text { of use; } \\
\mathrm{N}=\mathrm{TH} s \\
(105)\end{array}$} & \multirow[t]{2}{*}{ FL } & \multirow[t]{2}{*}{ UV } \\
\hline & & & & & & & & UM & $\%$ & & \\
\hline Anacardiaceae & $\begin{array}{l}\text { Sclerocarya birrea (A.Rich.) } \\
\text { Hochst. subsp. caffra (Sond.) }\end{array}$ & Morula/Mokano & Tree & Fruit & Fresh & $\begin{array}{l}\text { Juice is squeezed (raw), } \\
\text { dried and pounded. } \\
\text { Powder is poured in } \\
\text { the hot water. Steam } \\
\text { is inhaled (nasally) } \\
\text { under blanket. Thrice a } \\
\text { day }\end{array}$ & Rhinitis & 3 & 2.8 & 100 & 0.02 \\
\hline Apiaceae & *Daucus carota L. & Mokherotse & Herb & Tuber & Fresh & $\begin{array}{l}\text { Mixed with fresh root } \\
\text { of } M \text {. sapientum. } \\
\text { Macerated in cold } \\
\text { water for } 5 \text { hrs. Drop } \\
\text { of extract is applied } \\
\text { topically in the eyes. } \\
\text { Thrice a day }\end{array}$ & $\begin{array}{l}\text { Painful } \\
\text { eyes }\end{array}$ & 1 & 0.9 & 100 & 0.00 \\
\hline Apocynaceae & $\begin{array}{l}\text { Acokanthera rotundata } \\
\text { (Codd) Kupicha }\end{array}$ & Moethi & Shrub & Root & Dry & $\begin{array}{l}\text { Pounded and a pinch of } \\
\text { fingers is snuffed } \\
\text { (nasally). Thrice a day }\end{array}$ & Rhinitis & 10 & 9.5 & 100 & 0.09 \\
\hline Apocynaceae & $\begin{array}{l}\text { Strophanthus speciosus } \\
\text { (Ward \& Harv.) Reber }\end{array}$ & Morarwane & Shrub & Root & Dry & $\begin{array}{l}\text { Boiled for } 6-12 \\
\text { minutes. Extract is } \\
\text { taken orally. Thrice a } \\
\text { day }\end{array}$ & Fatigue & 23 & 21.9 & 100 & 0.21 \\
\hline \multirow[t]{2}{*}{ Araceae } & \multirow[t]{2}{*}{$\begin{array}{l}\text { Stylochaeton natalensis } \\
\text { Schott } \\
\text { Aloe spp. }\end{array}$} & \multirow[t]{2}{*}{ Mokunya/Mokušhete } & \multirow[t]{2}{*}{ Herb } & \multirow[t]{2}{*}{ Root } & \multirow[t]{2}{*}{ Dry } & $\begin{array}{l}\text { Boiled for } 5 \text { minutes. } \\
\text { Steam inhaled (nasally) } \\
\text { under blanket. Thrice a } \\
\text { day }\end{array}$ & Rhinitis & 102 & \multirow[t]{2}{*}{100} & \multirow[t]{2}{*}{100} & \multirow[t]{2}{*}{1} \\
\hline & & & & & & $\begin{array}{l}\text { Pounded and poured in } \\
\text { boiled water. Steam is } \\
\text { inhaled (nasally) under } \\
\text { blanket. Thrice a day }\end{array}$ & Rhinitis & 3 & & & \\
\hline Asphodelaceae & $\begin{array}{l}\text { Aloe marlothii A.Berger } \\
\text { subsp. Marlothii }\end{array}$ & $\begin{array}{l}\text { Thogo/ } \\
\text { Marobadibogale }\end{array}$ & Shrub & Leaf & Fresh & $\begin{array}{l}\text { Juice is squeezed (raw) } \\
\text { and a drop is applied } \\
\text { topically in the eyes. } \\
\text { Thrice a day }\end{array}$ & $\begin{array}{l}\text { Painful } \\
\text { eyes }\end{array}$ & 1 & 0.9 & 100 & 0.00 \\
\hline Asphodelaceae & $\begin{array}{l}\text { Artemisia afra Jacq. ex } \\
\text { Willd. var. afra }\end{array}$ & Sekgopha-sa-go-ema & Shrub & Leaf & Fresh & $\begin{array}{l}\text { Juice is squeezed (raw) } \\
\text { and a drop is applied } \\
\text { topically in the eyes. } \\
\text { Thrice a day }\end{array}$ & $\begin{array}{l}\text { Painful } \\
\text { eyes }\end{array}$ & 1 & 0.9 & 100 & 0.00 \\
\hline Asteraceae & Athrixia phylicoides DC & Legana/Moilanši & Herb & Leaf & Dry & $\begin{array}{l}\text { Boiled for } 4-5 \text { minutes. } \\
\text { Steam is inhaled } \\
\text { (nasally) under blanket. } \\
\text { Thrice a day }\end{array}$ & Rhinitis & 105 & 100 & 100 & 1 \\
\hline Asteraceae & $\begin{array}{l}\text { Geigeria burkei Harv. subsp. } \\
\text { burkei var. hirtella merxm }\end{array}$ & $\begin{array}{l}\text { Mohlahlaiša/ } \\
\text { Mmangwako }\end{array}$ & Shrub & Leaf & Dry & $\begin{array}{l}\text { Pounded and taken } \\
\text { orally with warm } \\
\text { water. Thrice a day }\end{array}$ & Rhinitis & 1 & 0.9 & 100 & 0.00 \\
\hline Asteraceae & Kleinia longiflora DC. & $\begin{array}{l}\text { Mmagae/ } \\
\text { Mmalefagong }\end{array}$ & Herb & $\begin{array}{l}\text { Whole } \\
\text { plant }\end{array}$ & Dry & $\begin{array}{l}\text { Pounded and mixed } \\
\text { with warm water. Drop } \\
\text { of extract is applied } \\
\text { topically in the eyes. } \\
\text { Thrice a day }\end{array}$ & $\begin{array}{l}\text { Painful } \\
\text { eyes }\end{array}$ & 26 & 24.7 & 100 & 0.24 \\
\hline Asteraceae & $\begin{array}{l}\text { *Schkuhria pinnata (Lam.) } \\
\text { Kuntze ex Thell. }\end{array}$ & Mmale & Shrub & Twig & Dry & $\begin{array}{l}\text { Pounded and mixed } \\
\text { with warm water. Drop } \\
\text { of extract is applied } \\
\text { topically in the eyes. } \\
\text { Thrice a day }\end{array}$ & $\begin{array}{l}\text { Painful } \\
\text { eyes }\end{array}$ & 50 & 47.6 & 100 & 0.47 \\
\hline Asteraceae & Senecio serratuloides DC. & $\begin{array}{l}\text { Šathume/Mošašane/ } \\
\text { Seralane }\end{array}$ & Herb & $\begin{array}{l}\text { Whole } \\
\text { plant }\end{array}$ & Fresh & $\begin{array}{l}\text { Macerated in cold } \\
\text { water for } 5-24 \text { hrs. } \\
\text { Drop of extract is } \\
\text { applied topically in the } \\
\text { eyes. Thrice a day }\end{array}$ & $\begin{array}{l}\text { Painful } \\
\text { eyes }\end{array}$ & 105 & 100 & 100 & 1 \\
\hline Asteraceae & $\begin{array}{l}\text { Vernonia natalensis Sch.Bip. } \\
\text { ex Walp. }\end{array}$ & Legatuludi & Shrub & Leaf & Dry & $\begin{array}{l}\text { Pounded and taken } \\
\text { orally with warm } \\
\text { water. Thrice a day }\end{array}$ & Fatigue & 3 & 2.8 & 100 & 0.02 \\
\hline Asteraceae & $\begin{array}{l}\text { Markhamia zanzibarica } \\
\text { (Bojer ex DC.) K.Schum }\end{array}$ & Mošuhla & Herb & Leaf & Dry & $\begin{array}{l}\text { Pounded and taken } \\
\text { orally with warm } \\
\text { water. Thrice a day }\end{array}$ & Headache & 1 & 0.9 & 100 & 0.00 \\
\hline Bignoniaceae & Commiphora marlothii Engl. & Bolebatša & Tree & Root & Dry & $\begin{array}{l}\text { Pounded and mixed } \\
\text { with warm water. Drop } \\
\text { of extract is applied } \\
\text { topically in the eyes. } \\
\text { Thrice a day }\end{array}$ & $\begin{array}{l}\text { Painful } \\
\text { eyes }\end{array}$ & 4 & 3.8 & 100 & 0.03 \\
\hline Burseraceae & $\begin{array}{l}\text { Warburgia salutaris } \\
\text { (G.Bertol.) Chiov. }\end{array}$ & Mphaphapha & Tree & Root & Dry & $\begin{array}{l}\text { Burned for about } 4 \\
\text { seconds. Smoke is } \\
\text { inhaled (nasally). Thrice } \\
\text { a day }\end{array}$ & Rhinitis & 1 & 0.9 & 100 & 0.03 \\
\hline
\end{tabular}




\begin{tabular}{|c|c|c|c|c|c|c|c|c|c|c|c|}
\hline \multirow[t]{3}{*}{ Canellaceae } & \multirow{3}{*}{$\begin{array}{l}\text { Capparis tomentosa Lam. } \\
\text { Cleome gynandra L. } \\
\text { Gymnosporia maranguensis } \\
\text { (Loes.) Loes. }\end{array}$} & \multirow[t]{3}{*}{ Molaka } & \multirow[t]{3}{*}{ Tree } & \multirow[t]{3}{*}{ Bark } & \multirow[t]{3}{*}{ Dry } & \multirow{3}{*}{$\begin{array}{l}\text { Boiled for } 4-11 \\
\text { minutes. Extract is } \\
\text { taken orally. Thrice a } \\
\text { day } \\
\text { Pounded and mixed } \\
\text { with dried powdered } \\
\text { root of } z \text {. capense. } \\
\text { Taken orally with warm } \\
\text { water. Thrice a day }\end{array}$} & Fatigue & 1 & 0.9 & 33.3 & \multirow[t]{3}{*}{0.02} \\
\hline & & & & & & & Headache & 1 & 0.9 & 33.3 & \\
\hline & & & & & & & Rhinitis & 1 & 0.9 & 33.3 & \\
\hline Capparaceae & $\begin{array}{l}\text { Gymnosporia senegalensis } \\
\text { (Lam.) Loes. }\end{array}$ & Moopatladi & Tree & Root & Dry & $\begin{array}{l}\text { Pounded and mixed } \\
\text { with cold water. Drop } \\
\text { of extract is applied } \\
\text { topically in the eyes. } \\
\text { Thrice a day }\end{array}$ & $\begin{array}{l}\text { Painful } \\
\text { eyes }\end{array}$ & 1 & 0.9 & 100 & 0.00 \\
\hline Capparaceae & $\begin{array}{l}\text { Commelina africana L. var. } \\
\text { Africana }\end{array}$ & Lerotho & Herb & Leaf & Dry & $\begin{array}{l}\text { Pounded and taken } \\
\text { orally with warm } \\
\text { water. Thrice a day }\end{array}$ & Rhinitis & 1 & 0.9 & 100 & 0.00 \\
\hline Celastraceae & $\begin{array}{l}\text { Kalanchoe brachyloba } \\
\text { Welw. ex Britten }\end{array}$ & Mokgoropo & Tree & Root & Dry & 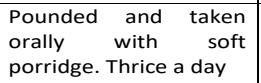 & Headache & 5 & 4.7 & 100 & 0.04 \\
\hline Celastraceae & Cyperus sexangularis Nees & Mphato & Tree & Root & Dry & $\begin{array}{l}\text { Pounded and mixed } \\
\text { with cold water. Drop } \\
\text { of extract is applied } \\
\text { topically in the eyes. } \\
\text { Thrice a day }\end{array}$ & $\begin{array}{l}\text { Painful } \\
\text { eyes }\end{array}$ & 4 & 3.8 & 100 & 0.03 \\
\hline Commelinaceae & $\begin{array}{l}\text { Euphorbia inaequilatera } \\
\text { Sond. var. inaequilatera }\end{array}$ & Bolebatša & Herb & Root & Dry & $\begin{array}{l}\text { Boiled for } 9-12 \\
\text { minutes. Drop of cold } \\
\text { extract is applied } \\
\text { topically in the eyes. } \\
\text { Thrice a day }\end{array}$ & $\begin{array}{l}\text { Painful } \\
\text { eyes }\end{array}$ & 1 & 0.9 & 100 & 0.00 \\
\hline Crassulaceae & Croton menyharthii Pax & $\begin{array}{l}\text { Moethi/ } \\
\text { Mošimanewanaga/ } \\
\text { Moritšikana }\end{array}$ & Shrub & Leaf & Fresh & $\begin{array}{l}\text { Rubbed (raw) between } \\
\text { hands and vapour is } \\
\text { inhaled (nasally). Thrice } \\
\text { a day }\end{array}$ & Headache & 105 & 100 & 100 & 1 \\
\hline \multirow[t]{2}{*}{ Cyperaceae } & \multirow[t]{2}{*}{$\begin{array}{l}\text { Euphorbia schinzii Pax } \\
\text { Jatropha zeyheri Sond. }\end{array}$} & \multirow[t]{2}{*}{ Mohlahla } & \multirow[t]{2}{*}{ Herb } & \multirow[t]{2}{*}{ Root } & \multirow[t]{2}{*}{ Dry } & $\begin{array}{l}\text { Pounded and taken } \\
\text { orally with warm } \\
\text { water. Thrice a day }\end{array}$ & Fatigue & 75 & \multirow[t]{2}{*}{72.3} & \multirow[t]{2}{*}{100} & \multirow[t]{2}{*}{0.72} \\
\hline & & & & & & $\begin{array}{l}\text { Pounded and } \\
\text { with powdered } \\
\text { root dried } \\
\text { orally } \text {. italica. Taken } \\
\text { porridge. Thrice a day } \\
\text { porth }\end{array}$ & Fatigue & 1 & & & \\
\hline \multirow[t]{2}{*}{ Euphorbiaceae } & \multirow[t]{2}{*}{$\begin{array}{l}\text { Spirostachys africana Sond. } \\
\text { Tragia dioica Sond. }\end{array}$} & \multirow[t]{2}{*}{$\begin{array}{l}\text { Kgamaswana/ } \\
\text { Mmatelaface }\end{array}$} & \multirow[t]{2}{*}{ Herb } & \multirow[t]{2}{*}{$\begin{array}{l}\text { Whole } \\
\text { plant }\end{array}$} & \multirow[t]{2}{*}{ Dry } & $\begin{array}{l}\text { Burned for about } 4-15 \\
\text { seconds. Smoke is } \\
\text { inhaled (nasally). Thrice } \\
\text { a day }\end{array}$ & Rhinitis & 2 & 1.9 & 66.6 & 0.01 \\
\hline & & & & & & $\begin{array}{l}\text { Pounded and mixed } \\
\text { with warm water. Drop } \\
\text { of extract is applied } \\
\text { topically in the eyes. } \\
\text { Thrice a day }\end{array}$ & $\begin{array}{l}\text { Painful } \\
\text { eyes }\end{array}$ & 1 & 0.9 & 33.3 & 0.00 \\
\hline Euphorbiaceae & $\begin{array}{l}\text { Senna italica Mill. subsp. } \\
\text { arachoides (Burch.) Lock }\end{array}$ & Moologa & Tree & Root & Dry & $\begin{array}{l}\text { Boiled for } 5 \text { minutes. } \\
\text { Steam is inhaled } \\
\text { (nasally) under blanket. } \\
\text { Thrice a day }\end{array}$ & $\begin{array}{l}\text { Painful } \\
\text { eyes }\end{array}$ & 2 & 1.9 & 100 & 0.01 \\
\hline \multirow[t]{2}{*}{ Euphorbiaceae } & \multirow{2}{*}{$\begin{array}{l}\text { Tylosema esculentum } \\
\text { (Burch.) A.Schreib. } \\
\text { Enicostema axillare (Lam.) } \\
\text { A.Raynal subsp. Axillare }\end{array}$} & \multirow[t]{2}{*}{ Ngaka-dianya } & \multirow[t]{2}{*}{ Herb } & \multirow[t]{2}{*}{ Root } & \multirow[t]{2}{*}{ Dry } & \multirow{2}{*}{$\begin{array}{l}\text { Boiled for 5-8 minutes. } \\
\text { Steam is inhaled } \\
\text { (nasally) under blanket. } \\
\text { Thrice a day }\end{array}$} & $\begin{array}{l}\text { Painful } \\
\text { eyes }\end{array}$ & 4 & 3.8 & 11.7 & \multirow[t]{2}{*}{0.33} \\
\hline & & & & & & & Rhinitis & 30 & 21.4 & 88.2 & \\
\hline Euphorbiaceae & $\begin{array}{l}\text { Clerodendrum ternatum } \\
\text { Schinz }\end{array}$ & Sephapabadiya & Herb & Root & Dry & $\begin{array}{l}\text { Boiled for } 5 \text { minutes. } \\
\text { Extract is taken orally. } \\
\text { Thrice a day }\end{array}$ & Fatigue & 1 & 0.9 & 100 & 0.00 \\
\hline Euphorbiaceae & $\begin{array}{l}\text { Cryptocarya transvaalensis } \\
\text { Burtt Davy }\end{array}$ & $\begin{array}{l}\text { Morekuri/ } \\
\text { Motampota }\end{array}$ & Tree & Root & Dry & $\begin{array}{l}\text { Burned for about } 7-8 \\
\text { minute. Smoke inhaled } \\
\text { (nasally). Thrice a day }\end{array}$ & Headache & 105 & 100 & 100 & 1 \\
\hline Euphorbiaceae & Adansonia digitata $\mathrm{L}$ & Bogopa/Mabatšane & Herb & Leaf & Dry & $\begin{array}{l}\text { Pounded and taken } \\
\text { orally with warm } \\
\text { water. Thrice day. }\end{array}$ & Fatigue & 1 & 0.9 & 100 & 0.00 \\
\hline Fabaceae & $\begin{array}{l}\text { Grewia bicolor Juss. var. } \\
\text { bicolor }\end{array}$ & Moroteladitšhoši & Herb & Root & Dry & $\begin{array}{l}\text { Pounded and mixed } \\
\text { with dried powered } \\
\text { root of } C \text {. sexangularis. } \\
\text { Taken orally with warm } \\
\text { water. Thrice a day }\end{array}$ & Fatigue & 1 & 0.9 & 100 & 0.00 \\
\hline Fabaceae & Grewia hispida Harv. & Monoga/Noga-koto & Herb & Root & Dry & $\begin{array}{l}\text { Pounded and taken } \\
\text { orally with warm } \\
\text { water. Thrice a day }\end{array}$ & Headache & 1 & 0.9 & 100 & 0.00 \\
\hline Gentianaceae & $\begin{array}{l}\text { Hibiscus meyeri Harv. subsp. } \\
\text { meyeri }\end{array}$ & $\begin{array}{l}\text { Makgonotšohle/ } \\
\text { Mphedu-ya-thaba }\end{array}$ & Herb & $\begin{array}{l}\text { Whole } \\
\text { plant }\end{array}$ & Dry & $\begin{array}{l}\text { Boiled for } 5 \text { minutes. } \\
\text { Steam is inhaled } \\
\text { (nasally) under blanket. } \\
\text { Thrice a day }\end{array}$ & Rhinitis & 105 & 100 & 100 & 1 \\
\hline Lamiaceae & Ficus burkei (Miq.) Miq. & Sebokane & Herb & $\begin{array}{l}\text { Whole } \\
\text { plant }\end{array}$ & Dry & $\begin{array}{l}\text { Boiled for } 5 \text { minutes. } \\
\text { Steam inhaled (nasally) } \\
\text { under blanket. Thrice a } \\
\text { day }\end{array}$ & Rhinitis & 105 & 100 & 100 & 1 \\
\hline
\end{tabular}




\begin{tabular}{|c|c|c|c|c|c|c|c|c|c|c|c|}
\hline Lauraceae & $\begin{array}{l}{ }^{*} \text { Moringa oleifera sensu } \\
\text { Exell \& Mendon }\end{array}$ & Kgosupsa & Tree & Bark & Dry & $\begin{array}{l}\text { Pounded and poured in } \\
\text { boiled water. Steam is } \\
\text { inhaled (nasally) under } \\
\text { blanket. Thrice a day }\end{array}$ & Rhinitis & 105 & 100 & 100 & 1 \\
\hline Malvaceae & ${ }^{*}$ Musa sapientum L & Mogoo & Tree & Root & Dry & $\begin{array}{l}\text { Boiled for } 6-10 \\
\text { minutes. Extract is } \\
\text { taken orally. Thrice a } \\
\text { day }\end{array}$ & Fatigue & 77 & 73.3 & 100 & 0.73 \\
\hline Malvaceae & $\begin{array}{l}\text { Myrothamnus flabellifolius } \\
\text { Welw. }\end{array}$ & Mogwane & Tree & Root & Dry & $\begin{array}{l}\text { Pounded and mixed } \\
\text { with cold water. Drop } \\
\text { of extract is applied } \\
\text { topically in the eyes. } \\
\text { Thrice a day }\end{array}$ & $\begin{array}{l}\text { Painful } \\
\text { eyes }\end{array}$ & 2 & 1.9 & 100 & 0.01 \\
\hline Malvaceae & ${ }^{*}$ Nymphaea mexicana Zucc. & $\begin{array}{l}\text { Mogwete/Mogolori/ } \\
\text { Lefielo }\end{array}$ & Shrub & Root & Dry & $\begin{array}{l}\text { Pounded and extract is } \\
\text { taken orally with warm } \\
\text { water. Thrice a day }\end{array}$ & $\begin{array}{l}\text { Painful } \\
\text { eyes }\end{array}$ & 1 & 0.9 & 100 & 0.00 \\
\hline Malvaceae & $\begin{array}{l}\text { Ximenia caffra Sond. var. } \\
\text { natalensis Sond. }\end{array}$ & $\begin{array}{l}\text { Motomelatšie/ } \\
\text { Mogatšatšipa/ } \\
\text { Motiatswane }\end{array}$ & Herb & Root & Dry & $\begin{array}{l}\text { Pounded and mixed } \\
\text { with cold water. Drop } \\
\text { of extract is applied } \\
\text { topically in the eyes. } \\
\text { Thrice a day }\end{array}$ & $\begin{array}{l}\text { Painful } \\
\text { eyes }\end{array}$ & 9 & 8.5 & 100 & 0.08 \\
\hline \multirow[t]{2}{*}{ Moraceae } & \multirow[t]{2}{*}{$\begin{array}{l}\text { Adenia spinosa Burtt Davy } \\
\text { Flueggea virosa (Roxb. ex } \\
\text { Willd.) Voigt subsp. Virosa }\end{array}$} & \multirow[t]{2}{*}{ Moumo } & \multirow[t]{2}{*}{ Tree } & \multirow[t]{2}{*}{ Fruit } & \multirow[t]{2}{*}{ Dry } & $\begin{array}{l}\text { Burned for about } 6 \\
\text { seconds. Smoke is } \\
\text { inhaled (nasally). Thrice } \\
\text { a day }\end{array}$ & Headache & 1 & \multirow[t]{2}{*}{1.9} & \multirow[t]{2}{*}{100} & \multirow[t]{2}{*}{0.01} \\
\hline & & & & & & $\begin{array}{l}\text { Boiled for } 5 \text { minutes. } \\
\text { Extract is taken orally. } \\
\text { Thrice a day }\end{array}$ & Headache & 1 & & & \\
\hline Moringaceae & $\begin{array}{l}\text { Sorghum bicolor (L.) } \\
\text { Moench subsp. } \\
\text { arundinaceum (Desv.) de } \\
\text { Wet \& Harlan }\end{array}$ & Moringka & Tree & Leaf & Dry & $\begin{array}{l}\text { Pounded and taken } \\
\text { orally with warm } \\
\text { water. Thrice a day }\end{array}$ & Fatigue & 61 & 58 & 100 & 0.58 \\
\hline Musaceae & *Zea mays subsp. mays L. & Mopanana & Tree & Root & Fresh & $\begin{array}{l}\text { Mixed with tuber of } D . \\
\text { carota. Macerated in } \\
\text { cold water for } 5 \text { hrs. } \\
\text { Drop of extract is } \\
\text { applied topically in the } \\
\text { eyes. Thrice a day }\end{array}$ & $\begin{array}{l}\text { Painful } \\
\text { eyes }\end{array}$ & 1 & 0.9 & 100 & 0.00 \\
\hline Myrothamnaceae & $\begin{array}{l}\text { Securidaca } \\
\text { longepedunculata Fresen. } \\
\text { var. longepedunculata }\end{array}$ & $\begin{array}{l}\text { Patše-ya-tšhwene/ } \\
\text { Matlapaneng }\end{array}$ & Herb & $\begin{array}{l}\text { Whole } \\
\text { plant }\end{array}$ & Dry & $\begin{array}{l}\text { Pounded and extract is } \\
\text { taken orally. Thrice a } \\
\text { day }\end{array}$ & Headache & 6 & 5.7 & 100 & 0.05 \\
\hline Nymphaeaceae & $\begin{array}{l}\text { Ptaeroxylon obliquum } \\
\text { (Thunb.) Radlk. }\end{array}$ & Hlapi & Herb & $\begin{array}{l}\text { Whole } \\
\text { plant }\end{array}$ & Dry & $\begin{array}{l}\text { Macerated in cold } \\
\text { water for } 30 \text { minutes to } \\
\text { an hour. Drop of } \\
\text { extract is applied } \\
\text { topically in the eyes. } \\
\text { Thrice a day }\end{array}$ & $\begin{array}{l}\text { Painful } \\
\text { eyes }\end{array}$ & 26 & 24.7 & 100 & 0.24 \\
\hline \multirow[t]{2}{*}{ Olacaceae } & \multirow{2}{*}{$\begin{array}{l}\text { Berchemia discolor } \\
\text { (Klotzsch) Hemsl. } \\
\text { Ziziphus mucronata Willd. } \\
\text { subsp. mucronata }\end{array}$} & \multirow[t]{2}{*}{ Motšhidi-kgomo } & \multirow[t]{2}{*}{ Tree } & \multirow[t]{2}{*}{ Root } & \multirow[t]{2}{*}{ Dry } & $\begin{array}{l}\text { Burned for about 5-11 } \\
\text { seconds. Smoke is } \\
\text { inhaled (nasally). Thrice } \\
\text { a day }\end{array}$ & Headache & 7 & \multirow[t]{2}{*}{7.6} & \multirow[t]{2}{*}{100} & \multirow[t]{2}{*}{0.07} \\
\hline & & & & & & $\begin{array}{l}\text { Pounded and mixed } \\
\text { with dried powdered } \\
\text { root of V. infausta. } \\
\text { Taken orally with warm } \\
\text { water. Thrice a day }\end{array}$ & Headache & 1 & & & \\
\hline \multirow[t]{3}{*}{ Passifloraceae } & \multirow{3}{*}{$\begin{array}{l}\text { Vangueria infausta Burch. } \\
\text { subsp. infausta } \\
\text { Zanthoxylum capense } \\
\text { (Thunb.) Harv. } \\
\text { Englerophytum } \\
\text { magalismontanum (Sond.) } \\
\text { T.D.Penn. }\end{array}$} & \multirow[t]{3}{*}{$\begin{array}{l}\text { Monna-apare/ } \\
\text { Pisayabatšumi/ } \\
\text { Mothema }\end{array}$} & \multirow[t]{3}{*}{ Shrub } & \multirow[t]{3}{*}{ Stem } & \multirow[t]{3}{*}{ Dry } & $\begin{array}{l}\text { Pounded and taken } \\
\text { orally with warm } \\
\text { water. Thrice a day }\end{array}$ & Fatigue & 26 & 24.7 & 53 & \multirow[t]{3}{*}{0.46} \\
\hline & & & & & & \multirow{2}{*}{$\begin{array}{l}\text { Boiled for } 5 \text { minutes. } \\
\text { Steam is inhaled } \\
\text { (nasally) under blanket. } \\
\text { Thrice a day }\end{array}$} & \begin{tabular}{|l|}
$\begin{array}{l}\text { Painful } \\
\text { eyes }\end{array}$ \\
\end{tabular} & 2 & 1.9 & 4 & \\
\hline & & & & & & & Rhinitis & 21 & 20 & 42.8 & \\
\hline Phyllanthaceae & $\begin{array}{l}{ }^{*} \text { Capsicum annuum L. var. } \\
\text { glabriusculum (Dunal) } \\
\text { Heiser \& Pickersgil }\end{array}$ & Mohlakaume & Shrub & Root & Dry & $\begin{array}{l}\text { Pounded and mixed } \\
\text { with cold water. Drop } \\
\text { of extract is applied } \\
\text { topically in the eyes. } \\
\text { Thrice a day }\end{array}$ & $\begin{array}{l}\text { Painful } \\
\text { eyes }\end{array}$ & 6 & 5.7 & 100 & 0.05 \\
\hline Poaceae & *Nicotiana tabacum L. & Mabele-thoro & Herb & Seed & Dry & $\begin{array}{l}\text { Pounded and taken } \\
\text { orally with Mageu } \\
\text { drink or soft porridge. } \\
\text { Thrice a day }\end{array}$ & Fatigue & 18 & 17.1 & 100 & 0.17 \\
\hline Poaceae & $\begin{array}{l}\text { Solanum panduriforme } \\
\text { E.Mey. }\end{array}$ & Mabele & Herb & Root & Dry & $\begin{array}{l}\text { Pounded and taken } \\
\text { orally with warm } \\
\text { water. Thrice a day }\end{array}$ & Headache & 1 & 0.9 & 100 & 0.00 \\
\hline \multirow[t]{3}{*}{ Polygalaceae } & \multirow{3}{*}{$\begin{array}{l}\text { Lasiosiphon caffer Meisn. } \\
\text { Xerophyta retinervis Baker } \\
\text { Lippia javanica (Burm.f.) } \\
\text { Spreng. }\end{array}$} & \multirow[t]{3}{*}{$\begin{array}{l}\text { Mphesu/ } \\
\text { Mpitlamarago }\end{array}$} & \multirow[t]{3}{*}{ Tree } & \multirow[t]{3}{*}{ Root } & \multirow[t]{3}{*}{ Dry } & $\begin{array}{l}\text { Boiled for } 5-10 \\
\text { minutes. Steam is } \\
\text { inhaled (nasally) under } \\
\text { blanket. Thrice a day }\end{array}$ & Headache & 105 & 100 & 98.1 & 1.01 \\
\hline & & & & & & $\begin{array}{l}\text { Pounded and taken } \\
\text { orally with warm } \\
\text { water. Thrice a day }\end{array}$ & Rhinitis & 1 & 1.4 & 1.8 & \\
\hline & & & & & & $\begin{array}{l}\text { Pounded and mixed } \\
\text { with powdered root of } \\
z \text {. capense. A pinch of } \\
\text { finger is snuffed } \\
\text { (nasally). Thrice a day }\end{array}$ & Rhinitis & 1 & & & \\
\hline
\end{tabular}




\begin{tabular}{|c|c|c|c|c|c|c|c|c|c|c|c|}
\hline \multirow[t]{2}{*}{ Ptaeroxylaceae } & \multirow[t]{2}{*}{$\begin{array}{l}\text { Siphonochilus aethiopicus } \\
\text { (Schweinf.) B.L.Burtt } \\
\text { Sclerocarya birrea (A.Rich.) } \\
\text { Hochst. subsp. caffra (Sond.) }\end{array}$} & \multirow[t]{2}{*}{ Mogabaletswana } & \multirow[t]{2}{*}{ Tree } & \multirow[t]{2}{*}{ Root } & \multirow[t]{2}{*}{ Dry } & $\begin{array}{l}\text { Burned for about 5-11 } \\
\text { minutes. Smoke is } \\
\text { inhaled (nasally). Thrice } \\
\text { a day }\end{array}$ & Headache & 1 & \multirow[t]{2}{*}{90.4} & \multirow[t]{2}{*}{100} & \multirow[t]{2}{*}{0.90} \\
\hline & & & & & & $\begin{array}{l}\text { Pounded and taken } \\
\text { orally with warm } \\
\text { water. Thrice a day }\end{array}$ & Headache & 94 & & & \\
\hline Rhamnaceae & *Daucus carota $\mathrm{L}$. & $\begin{array}{l}\text { Moneyee/ } \\
\text { Mogokgoma }\end{array}$ & Tree & Root & Dry & $\begin{array}{l}\text { Pounded and taken } \\
\text { orally with warm } \\
\text { water. Thrice a day }\end{array}$ & $\begin{array}{l}\text { Painful } \\
\text { eyes }\end{array}$ & 12 & 11.4 & 100 & 0.11 \\
\hline Rhamnaceae & $\begin{array}{l}\text { Acokanthera rotundata } \\
\text { (Codd) Kupicha }\end{array}$ & Mokgalo & Tree & Root & Dry & $\begin{array}{l}\text { Pounded and taken } \\
\text { orally with warm } \\
\text { water. Thrice a day }\end{array}$ & Headache & 1 & 0.9 & 100 & 0.00 \\
\hline \multirow[t]{2}{*}{ Rubiaceae } & \multirow[t]{2}{*}{$\begin{array}{l}\text { Strophanthus speciosus } \\
\text { (Ward \& Harv.) Reber } \\
\text { Stylochaeton natalensis } \\
\text { Schott }\end{array}$} & \multirow[t]{2}{*}{ Mmilo } & \multirow[t]{2}{*}{ Tree } & \multirow[t]{2}{*}{ Root } & \multirow[t]{2}{*}{ Dry } & $\begin{array}{l}\text { Pounded and mixed } \\
\text { with dried powdered } \\
\text { root of } X \text {. caffra. Taken } \\
\text { orally with warm } \\
\text { water. Thrice a day }\end{array}$ & Headache & 1 & 0.9 & \multirow[t]{2}{*}{100} & \multirow[t]{2}{*}{0.09} \\
\hline & & & & & & $\begin{array}{l}\text { Pounded and taken } \\
\text { orally with warm } \\
\text { water. Thrice a day }\end{array}$ & Headache & 9 & 8.5 & & \\
\hline \multirow[t]{4}{*}{ Rutaceae } & \multirow{4}{*}{$\begin{array}{l}\text { Aloe spp. } \\
\text { Aloe marlothii A.Berger } \\
\text { subsp. Marlothii } \\
\text { Artemisia afra Jacq. ex } \\
\text { Willd. var. afra } \\
\text { Athrixia phylicoides DC }\end{array}$} & \multirow[t]{4}{*}{ Monokwane/ Moregakgaka } & \multirow[t]{4}{*}{ Tree } & \multirow[t]{4}{*}{ Root } & \multirow[t]{4}{*}{ Dry } & $\begin{array}{l}\text { Pounded and taken } \\
\text { orally with warm } \\
\text { water. Thrice a day }\end{array}$ & Headache & 26 & 24.7 & 19.8 & \multirow[t]{4}{*}{1.24} \\
\hline & & & & & & $\begin{array}{l}\text { Pounded and mixed } \\
\text { with dried powdered } \\
\text { bark of } W \text {. salutaris. } \\
\text { Taken orally with warm } \\
\text { water. Thrice a day }\end{array}$ & Rhinitis & 1 & 100 & 80.1 & \\
\hline & & & & & & 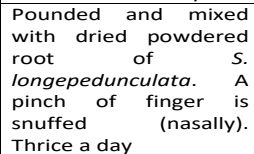 & Rhinitis & 1 & & & \\
\hline & & & & & & $\begin{array}{l}\text { Boiled for } 5-8 \text { minutes. } \\
\text { Steam is inhaled } \\
\text { (nasally) under blanket. } \\
\text { Thrice a day }\end{array}$ & Rhinitis & 103 & & & \\
\hline Sapotaceae & $\begin{array}{l}\text { Geigeria burkei Harv. subsp. } \\
\text { burkei var. hirtella merxm }\end{array}$ & Mohlatshwa & Tree & Root & Dry & $\begin{array}{l}\text { Pounded and mixed } \\
\text { with cold water. Drop } \\
\text { of extract is applied } \\
\text { topically in the eyes. } \\
\text { Thrice a day }\end{array}$ & $\begin{array}{l}\text { Painful } \\
\text { eyes }\end{array}$ & 26 & 24.7 & 100 & 0.24 \\
\hline Solanaceae & Kleinia longiflora DC. & Mophepha & Shrub & Root & Dry & $\begin{array}{l}\text { Pounded and taken } \\
\text { orally with warm } \\
\text { water. Thrice a day }\end{array}$ & Headache & 2 & 1.9 & 100 & 0.01 \\
\hline \multirow[t]{2}{*}{ Solanaceae } & \multirow{2}{*}{$\begin{array}{l}\text { *Schkuhria pinnata (Lam.) } \\
\text { Kuntze ex Thell. } \\
\text { Senecio serratuloides DC. }\end{array}$} & \multirow[t]{2}{*}{ Mofola } & \multirow[t]{2}{*}{ Shrub } & \multirow[t]{2}{*}{ Leaf } & \multirow[t]{2}{*}{ Dry } & \multirow{2}{*}{$\begin{array}{l}\text { Pounded and a pinch of } \\
\text { a finger is snuff } \\
\text { (nasally). Thrice a day }\end{array}$} & Headache & 2 & 1.9 & 7.6 & 0.24 \\
\hline & & & & & & & Rhinitis & 24 & 22.8 & 92.3 & \\
\hline Solanaceae & $\begin{array}{l}\text { Vernonia natalensis Sch. Bip. } \\
\text { e x Walp. }\end{array}$ & Mothola-o-moserolwane & Herb & Root & Dry & $\begin{array}{l}\text { Pounded and taken } \\
\text { orally with warm } \\
\text { water. Thrice a day }\end{array}$ & Headache & 17 & 16.1 & 100 & 0.16 \\
\hline Thymelaeacea & $\begin{array}{l}\text { Markhamia zanzibarica } \\
\text { (Bojer ex DC.) K.Schum }\end{array}$ & Nkekologe & Shrub & Root & Dry & $\begin{array}{l}\text { Pounded and poured in } \\
\text { boiled water. Steam is } \\
\text { inhaled (nasally) under } \\
\text { blanket. Thrice a day }\end{array}$ & Rhinitis & 105 & 100 & 100 & 1 \\
\hline Velloziaceae & Commiphora marlothii Engl. & Thuse & Herb & Root & Dry & $\begin{array}{l}\text { Pounded and taken } \\
\text { orally with warm } \\
\text { water. Thrice a day }\end{array}$ & Headache & 17 & 16.1 & 100 & 0.16 \\
\hline Verbenaceae & $\begin{array}{l}\text { Warburgia salutaris } \\
\text { (G.Bertol.) Chiov. }\end{array}$ & $\begin{array}{l}\text { Mošunkwane/ } \\
\text { motlaba-dipoo }\end{array}$ & Shrub & Leaf & Fresh & $\begin{array}{l}\text { Boiled for } 5-10 \\
\text { minutes. Steam is } \\
\text { inhaled (nasally) under } \\
\text { blanket. Thrice a day }\end{array}$ & Rhinitis & 105 & 100 & 100 & 1 \\
\hline Zingiberaceae & Capparis tomentosa Lam. & Serokolo & Herb & Bulb & Fresh & $\begin{array}{l}\text { Raw piece is rubbed } \\
\text { between hands and } \\
\text { vapour inhaled } \\
\text { (nasally). Thrice a day }\end{array}$ & Rhinitis & 25 & 23.8 & 100 & 0.23 \\
\hline
\end{tabular}




\section{The use of plants to treat ailments}

Further analysis of the finding from this study showed that of 63 plant species recorded, 20.6\% (n=13); $A$. afra, Acokanthera rotundata, Athrixia phylicoides, C. ternatum, Cleome gynandra, Aloe marlothii, C. transvaalensis, E. axillare, L. javanica, L. caffer, Siphonochilus aethiopicus, Sclerocarya birrea, and $S$. natalensis were used exclusively as rhinitis treatment, and $11.1 \%(\mathrm{n}=7)$ of plants namely Adenia spinosa and Warburgia salutaris (fatigue and painful eyes), Euphorbia inaequilatera and Euphorbia schinzii (rbinitis and painful eyes), Nicotiana tabacum (headache), S. longepedunculata (headache) and Z. capense (headache) used by Bapedi THs for rhinitis and the mentioned perceived symptoms. The reminder of the plants $(68.2 \%, n=43)$ were used wholly as remedies for the following symptoms; fatigue, headache and painful eyes in patients diagnosed with rhinitis (Table 1).

\section{Plant habit}

Growth forms of the 63 documented plants in this study were analysed. Accordingly, herbs $(39.6 \%, \mathrm{n}=25)$ were the most common habit used for medicinal purposes, followed by trees $(36.5 \%, \mathrm{n}=23)$ and shrubs $(23.8 \%, \mathrm{n}=15)$ respectively.

\section{Fidelity level (FL) and use value (UV)}

Of all $(n=63)$ the medicinal plants documented in this study, $A$. afra (UM=105 and $\mathrm{FL}=100$; rhinitis), C. ternatum (UM=105 and $\mathrm{FL}=100$; rhinitis), $C$. transvaalensis (UM=105 and $\mathrm{FL}=100$; rhinitis), E. axillare (UM=105 and $\mathrm{FL}=100$; rhinitis), $K$. bracbyloba (UM=105 and $\mathrm{FL}=100$; headache), L. caffer $(\mathrm{UM}=105$ and $\mathrm{FL}=100$; rhinitis), L. javanica (UM=105 and $\mathrm{FL}=100$; rhinitis), $S$. pinnata (UM=105 and FL=100; painful eyes), S. longepedunculata (UM=105 and $\mathrm{FL}=100$; headache), S. africana (UM=105and $\mathrm{FL}=100$; headache), $S$. natalensis (UM=105 and $\mathrm{FL}=100$; rhinitis), and $Z$. capense (UM=105 and $\mathrm{FL}=80.1$; rhinitis, and $\mathrm{UM}=26$ and $\mathrm{FL}=19.8$; headache), respectively had the highest FL as treatments of the mentioned ailment/s.

The highest UV calculated in this study was 1.24 for $Z$. capense (headache and rhinitis therapies), and 1.01 for $S$. longepedunculata (headache and rhinitis), respectively. This was closely and equally followed by $A$. afra, C. ternatum, C. transvaalensis, E. axillare, L. caffer, L. javanica, S. natalensis (UV=1; rhinitis, for each), $K$. brachyloba and $S$. africana (UV=1; headache, for each) as well as $S$. pinnata (UV=1; painful eyes).

African Health Sciences Vol 18 Issue 4, December, 2018

\section{Plant parts used, mode of preparations, dosages and} administrations

Root $(55.5 \%, \mathrm{n}=35)$, followed by leaf $(19 \%, \mathrm{n}=12)$, whole plant $(11.1 \%, n=7)$, bark and fruit $(3.1 \%, n=2$, for each), bulb, seed, tuber, twig and stem $(1.5 \%, \mathrm{n}=1$, for each), respectively were mostly used plant parts in this study. Larger number of these plant parts was prepared by THs in their dry $(85.7 \%, \mathrm{n}=54)$ state than when they are still fresh $(14.2 \%, \mathrm{n}=9)$.

Overall, 77 recipes prepared from the recorded plant parts were documented as described by Bapedi THs (Table 1). About $87 \%(n=67)$ of these recipes was made from a single plant part, and just $12.9 \%(n=10)$ was prepared from mixtures of diverse plant parts. The most common methods of preparation of the 77 documented recipes described by Bapedi THs were pounding (59.7\%, $\mathrm{n}=46)$, boiling $(20.7 \%, \mathrm{n}=16)$, burning $(7.7 \%, \mathrm{n}=6)$, macerating $(5.1 \%, \mathrm{n}=4)$, squeezing and rubbing $(2.5 \%$, $\mathrm{n}=2$, for each), squeezing and pounding $(1.2 \%, \mathrm{n}=1)$, respectively. Most of the prepared recipes/remedies in this study were dispensed orally $(44.1 \%, \mathrm{n}=34)$, nasally $(33.7 \%, n=26)$ and topically $(22 \%, n=17)$, respectively. Oral medication was prescribed as liquid or powder ( $\mathrm{Ta}$ ble 1). Pounded therapies were mainly taken with warm water as a vehicle. However, in few instance these therapies were mixed with additives such as Mageu ${ }^{\circledR}$ drink or soft porridge. According to THs these additives enhance the effectiveness of prescriptions. Nasal remedies were primarily taken as steam under a blanket.

Dosage strength of all herbal preparation documented in the present study was determined. In general, consistencies amongst THs with regards to this were only noted for boiled liquid medicine taken orally. For instance, a metal cup $(500 \mathrm{ml})$ was prescribed by all Bapedi THs three times a day (morning,midday and evening). However, dosage regimes of the rest of the herbal recipes made by these THs was not standardised, and depended on an individual healers' experience.

\section{Discussion}

To the best of our knowledge, the present study is the first in South Africa and Africa as a continent to investigate plants used traditionally by THs or lay people to treat rhinitis and related symptoms, thus there is a dearth of comparative data against which to evaluate our findings. As a matter of fact, there is generally a scanty of information regarding the use of herbal remedies to treat these 
conditions worldwide. However, few attempts to address this gap in knowledge have been made in countries such as Turkey ${ }^{15}$ and India ${ }^{16}$. Comparison of medicinal plants documented in these two studies with ours is divergent, probably due to the fact that diverse cultures of the world possess their own specific knowledge of plant use and tradition $^{21}$. We documented 63 plant species belonging to 40 botanical families, mainly the Asteraceae, Euphorbiaceae and Malvaceae used by Bapedi THs to treat rhinitis and related symptoms. Kanakavalli et al. ${ }^{16}$ reported the dominant of Fabaceae, and Sayin et al. ${ }^{15}$ found that all the botanical families in their study were each equally represented by a single species. The dominance of Asteracea, Malvaceae and Euphorbiaceae in our study could be attributed to their wider distribution across the studied sites, which in turn afforded local THs with higher diversity of plants to explore healing potential, and subsequently experiment against various human ailments including rhinitis. The remaining families recorded in this study were represented with less than three species used to heal rhinitis and related symptoms, thus correspondingly suggesting that their members are less popular amongst the interviewed Bapedi THs as treatment of these diseases. Overall higher number of taxa and associated botanical families documented in this study shows that Bapedi THs are knowledgeable about diversity of plants useful for rhinitis and related conditions. Also such high diversity to some extent is a good indicator that the studied areas harbour diverse habitats which supports a rich curative floristic wealth useful for these illnesses.

The widespread utilisations of species such as $A$. afra, $C$. ternatum, C. transvaalensis, E. axillare, K. brachyloba, L. caffer, L. javanica, S. pinnata, S. longepedunculata, S. africana, S. natalensis and $Z$. capense in this study emphasize their importance as remedies in Bapedi traditional healing sector, and also demonstrate that there are vital ethnobotanical contact points among THs in the studied districts. However, the extent of utilisation of theremaining species in this study is also understandable given that native knowledge is not uniformly distributed amongst culturally homogeneous group living in the same and different biophysical territory ${ }^{22}$, and that healers have different knowledge regarding herbal medicine used for human ailments.

To the best of our knowledge all the species ( $A$. afra, $A$. rotundata, $A$. phylicoides, $C$. ternatum, $C$. gynandra, Commipho- ra marlothii, C. transvaalensis, E. axillare, L. javanica, L. caffer, S. aethiopicus, $S$. birrea, and $S$. natalensis) used exclusively as cure for rhinitis are recorded for the first time in the present study as remedies for rhinitis, probably due to lack of African ethnobotanical studies focusing on this affliction. Overall, $A$. afra, C. ternatum, C. transvaalensis, E. axillare, L. caffer, L. javanica and S. natalensis was important species used by all THs $(n=105)$ who treated this respiratory infection. Although not exclusively used for rhinitis, traditional utilisation of some of these species particularly $A$. afra ${ }^{23}$ and $L$. javanica ${ }^{24}$ against various respiratory infections in general are supported by scientific studies; hence they are mostly utilized by Bapedi THs. To yield more critical insights into the potential of most of the above-widely used taxa in this study, as future treatment of rhinitis, and to better understand rationales as to why Bapedi THs prefer them, an investigation in to their effectiveness and safety specifically in the treatment of rhinitis should be investigated.

Amongst the $11.1 \%$ of plants namely $A$. spinosa and $W$. salutaris (fatigue and painful eyes), E. inaequilatera and E. schinzii (rhinitis and painful eyes), N. tabacum (headache) and $Z$. capense (rhinitis and headache) used by Bapedi THs for rhinitis and the mentioned perceived symptoms, only use of $N$. tabacum $^{25}$ as well as $S$. longepedunculata ${ }^{26}$ to treat headache was reported in literature. Thus uses of both these species for rhinitis are recorded in our study for the first time. Similarly, medicinal use of the remaining plants A. spinosa, E. inaequilatera, E. schinzii, $W$. salutaris and $Z$. capense for rhinitis and the aforesaid ailment/s are so far restricted to Bapedi THs.

Similarly, larger number of species recorded in this study as being used by THs to heal fatigue, headache and painful eyes (table 1), in patients diagnosed with rhinitis are recorded for the first time as medicines for these conditions. However, some of the medical application/s of Adansonia digitata ${ }^{27}$, Capsicum annum ${ }^{28}$, C. gynandra ${ }^{29}$, Daucus carota $^{30}$, Myrothamnus flabellifolius ${ }^{31}$, Moringa oleifera ${ }^{32}$, Ptaeroxylon obliquum ${ }^{33}, S$. africana ${ }^{34}$, S. pinnata ${ }^{35}$, Vernonia natalensis ${ }^{36}$, Ximenia caffra ${ }^{37}, Z e a$ mays ${ }^{38}$ and Ziziphus mucrona$t a^{39}$ by Bapedi THs are in partial agreements with previous studies conducted in other areas of South Africa, African countries and elsewhere, which reported applications of these species for fatigue, headache or painful eyes. in rhinitis suffers with various medicinal plants by Bapedi THs 
is an indication that they do take this respiratory infection serious, and thus investigation of the efficacy of these plants should be conducted. Effective herbal treatments of these symptoms are equally imperative in achieving the best outcomes of diagnosed patients. This is because it might assist in eliminating both occurrences and associated health impact of such symptoms that might affect quality.

Our study revealed that THs mainly prefer herbaceous and tree species for the preparation of herbal medicine to heal and manage rhinitis and related symptoms. Their distinct preferences of herbaceous species could be attributed to the fact that they are abundant at close proximity and are easy to harvest with less effort, both compared to trees and shrubs. On the other hand, utilisation of these two growth forms might be due to their ease of identification in the wild, high adaptability as well as capacity to proliferate in various seasons, thus available throughout the year. In general, the above recorded habits might be a reflection of the curative plant community structure across the studied areas.

Plant species which scored the highest FL as treatments of rhinitis and related symptoms in this study indicate greater agreement among the THs regarding their phyto-therapeutic use/s which might be attributed to efficacy, hence their strong selectivity over the others. Thus, such species should be prioritised for phytochemistry and pharmacological properties studies. Similar attention must be given to species such as $Z$. capense (headache and rhinitis therapies), $S$. longepedunculata (headache and rhinitis), A. afra, C. ternatum, C. transvaalensis, E. axillare, $L$. caffer, L. javanica, S. natalensis (rhinitis), K. brachyloba and $S$. africana (headache, for each) and $S$. pinnata (painful eyes) with the highest UV index. However, the high UVs of these species also indicates the need to promote their sustainable utilisations and harvesting.

Roots and leaves were mostly used plant parts in this study. On contrary, Kanakavalli et al. ${ }^{16}$ reported leaf, rhizome and whole plant as equally favoured medicinal plant parts for preparation of rhinitis remedies. The observed discrepancies might be due to the larger number $(n=63)$ of plant recorded in our study compared to just nine species noted by this authors. Larger number of plant parts in our study was prepared by THs in their dry) state than when they are still fresh, thus suggesting that few species are grown in home gardens or available in close vicinity for instance use as medicines. Not surprising, about $87 \%$ of herbal recipes was made from a single plant part, perhaps due to the ease of preparation compared to poly-recipes. Overall, diverse procedures of herbal preparing medicines recorded in this study might be attributed to THs' preferences or is a reflection of an old tradition amid these THs observed from various mentors. Different route of administering remedies such as burning and inhalation, rubbing between hands for inhalation of vapour and snuffing might be effective for treatment and management of rhinitis, mainly due to the fact that prescriptions go directly to the nasal membranes, thus might act on various components of the nasal inflammatory process reducing inflammatory cell numbers.

\section{Conclusion}

Our study is the first to document plants used traditionally to treat rhinitis and related symptoms in South Africa and Africa at large. Therefore, it is not surprising that all species used by interviewed Bapedi THs are recorded in this survey for the first time as rhinitis cure. On the other hand, high usage of some of the plant species implicated by these THs in the treatments of symptoms they perceived as allied to rhinitis across the studied district and municipalities, confirm the authenticity of data gathered and importance of such species. Overall, the medicated claims of the species inventoried in the study need to be evaluated through phytochemical and pharmacological investigations. Scanty of ethnobotanical information focusing on treatment of rhinitis and related symptoms in South Africa and Africa as a continent call for an urgent need to record such valuable data before it becomes inaccessible or vanishes together with its holders.

\section{Acknowledgements}

We are thankful to the THs practicing in the Capricorn, Sekhukhune and Waterberg districts (Limpopo Province), for sharing their ethnobotanical knowledge on plants used to treat rhinitis. This study was funded by the South African National Research foundation (NRF).

\section{Conflict of interest}

None declared.

\section{References}

1. Green RJ, Hockman M, Friedman R, Davis M, McDonald M, Seedat R, Levin M, Potter P, Feldman C. Chronic 
rhinitis in South Africa: Update 2013. South African Medical Journal. 2013; 103: 419-422.

2. Bousquet J, Dahl R, Khaltaev N. Global alliance against chronic respiratory diseases. Allergy. 2007; 62: 216-223.

3. Dykewicz, M.S., Fineman, S., Skoner, D.P., Nicklas, R, Lee, R. Diagnosis and management of rhinitis: Complete guidelines of the joint task force on practice parameters in allergy, asthma, and immunology. Annals of Allergy, Astbma and Immunology. 1998; 81: 478-518.

4. Morais-Almeida M, Pite H, Pereira AM, Todo-Bom A, Nunes C, Bousquets J, Fonseca J. Prevalence and classification of rhinitis in the elderly: A nationwide survey in Portugal. Allergy. 2013; 68: 1150- PubMed ;1157.

5. Eriksson J. Prevalence, risk factors and comorbidity of rhinitis, asthma and aspirin intolerance in West Sweden. University of Gothenburg, Sweden, 2012.

6. Desalu OO, Salami AK, Iseh KR, Oluboyo O. Prevalence of self-reported allergic rhinitis and its relationship with asthma among adult Nigerians. Journal of Investigational Allergology and Clinical Immunology. 2009; 19: 474-80.

7. Pefura-Yone EW, Kengne AP, Balkissou AD. Prevalence of asthma and allergic rhinitis among adults in Yaounde, Cameroon. PLoS. 2015; 10: 4: Doi:10.1371/ journal.pone.0123099.

8. Georgy V, Fahim HI, El-Gaafary M, Walters S. Prevalence and socioeconomic associations of asthma and allergic rhinitis in northern Africa. European Respiratory Journal. 2006; 28: 756-762.

9. Zar HJ, Ehrlich RI, Workman L, Weinberg EG. The changing prevalence of asthma, allergic rhinitis and atopic eczema in African adolescents from 1995 to 2002. Pediatric Allergy and Immunology. 2007; 18: 560-565.

10. Butler N. National Guidelines at a glance: Allergic rhinitis. SA Pharmacentical Journal. 2009; 48: 25-27.

11. Montoro J, Sastre J, Jáuregui I, Bartra J, Dávila I, Cuvillo AD, Ferrer M, Mullol J, Valero A. Allergic rhinitis: Continuous or on demand antihistamine therapy? Journal of Investigational Allergology and Clinical Immunology. 2007; 17: 21-27.

12. Van Cauwenberge P, Bachert C, Passalacqua G, Bousquet J, Canonica GW, Durham SR, Fokkens WJ, Howarth PH, Lund HJ, Malling N, Mygind N, Passali D, Scadding GK, Wang DY. Consensus statement on the treatment of allergic rhinitis. Allergy. 2000; 55:116-134.

13. DeGuzman DA, Bettcher CM, Van Harrison R, Holland CL, Reed LM, Remington TM, Zacharek MA. Allergic rhinitis. University of Michigan, Michigan, United States, 2013.
14. Licari A, Ciprandi G, Marseglia A, Castagnoli R, Barberi S, Caimmi S, Marseglia GL. Current recommendations and emerging options for the treatment of allergic rhinitis. Expert Review of Clinical Immunology. 2014; 1: 1-11. 15. Sayin I, Cingi C, Oghan O, Baykal B, Ulusoy S. Complementary therapies in allergic rhinitis. Allergy. Available from: Http://dx.doi.org/10.1155/2013/938751. Site accessed on 01.12.2017, 2013.

16. Kanakavalli K, Parthibhan P, Geetha V, Chithradevi $\mathrm{R}$, Latharani M, Thillaivanan. S. A review on anti-allergic herbs in Siddha system of medicine. Journal of Pharmaceutical Research and Bio-Science. 2014; 3: 312-320.

17. Elujoba AA, Odeleye OM, Ogunyemi CM (2005). Traditional medicine development for medical and dental primary health care delivery system in Africa. African Journal of Traditional, Complementary and Alternative Medicines. 2005; 2: 41-61.

18. Statistics South Africa. Census 2011 Census in brief. Statistics South Africa, Pretoria, 2011b.

19. Al-Quran S. Ethnopharmacological survey of wild medicinal plants in Showbak, Jordan. Journal of Ethnopharmacology. 2009; 123: 45-50.

20. Phillips OL, Gentry AH. The useful plants of Tambopata, Peru: II additional hypotheses testing in quantitative ethnobotany. Economic Bototant. 1993; 47: 33-43.

21. Cotton CM. Ethno botany: Principles and Applications. John Wiley and Sons, New York, 1996.

22. Thomas Y. Negotiating difference: Discourses of indigenous knowledge and development in Ghana. Political and legal anthropology review. 2008; 31: 224-242.

23. Patil GV, Dass SK, Chandra R. Artemisia afra and modern diseases. Journal of Pharmacognosy. 2011; 2: 105. Doi: 10.4172/2153-0645.1000105.

24. York T, Van Vuuren SF, De wet H. An antimicrobial evaluation of plants used for the treatment of respiratory infections in rural Maputaland, KwaZulu-Natal, South Africa. Journal of Ethnopharmacology. 2012; 144:118-127.

25. Charlton A. Medicinal uses of tobacco in history. Journal of the Royal Society of Medicine. 2004; 97: 292-296.

26. Mustapha, A.A. Ethno-medico-botanical uses of $S_{e-}$ curidaca longepedunculata Fresen (Family-Polygalaceae) from the Local Government, Nasarawa State, Nigeria. Journal of Natural Remedies. 2013; 13: 134-137.

27. Wickens GE. The uses of the baobab (Adansonia digitata L.) in Africa. In: Taxonomic aspects of African economic botany (Kunkel, G. Ed.). A.E.T.F.A.T. Las Palmas de Gran Canaria, 1979.

28. Shai-Srivastava S, Csere, A, Andrew, J. Alternative

African Health Sciences Vol 18 Issue 4, December, 2018 
medicine in chronic migraine 2014: What clinicians need to know. Available from: Http://www.practicalpainmanagement.com. site accessed on 12.12.2017, 2016. 29. Mishra SS, Moharana SK, Dash MR. Review on Cleome gynandra. International Journal of Research in Pharmacy and Chemistry. 1: 681-689, 2011.

30. Sandhu PS, Singh B, Gupta V, Bansal P, Kumar D. Potential herbs used in ocular diseases. International Journal of Pharmacentical Sciences Review and Research. 2010; 3: 1127-1140.

31. Bio-innovation Zimbabwe. Myrothamnus flabellifolia: Resurrection-Bush. Available from: Http://bio-innovation.org/work/resurrection-bush/. Site accessed on 12.09.2017, 2016.

32. Mehta J, Shukla A, Bukhariya V, Charde R. The magic remedy of Moringa oleifera: An overview. International Journal of Biomedical and Advance Research. 2: 215-227.

33. Schirmer P. The Concise Illustrated South African Encyclopedia. Central News Agency Ltd, 1980.
34. Van Wyk B, Oudshoorn V, Gericke N. Medicinal plants of South Africa. Briza Publications, Pretoria, 1997. 35. Moteetee A, Van Wyk, B-E. The medical ethnobotany of Lesotho: A review. Bothalia. 2011; 41: 209- 228. PubMed

36. Plantzafrica. Vernonia natalensis Sch.Bip. ex Walp. Available from: Http://www.plantzafrica.com/planttuv/ vernonnat.htm. Site accessed on 12.11.2017, 2016.

37. Arbonnier M. Trees, shrubs and lianas of West African dry zone. Margraf Publisher, Mnhn, 2004.

38. Sahani S, Mall TP. Diversity of potent ethno-medicinal flora for headache from North-Tarai Forests of (U. P.) India. International Journal of Interdisciplinary and Multidisciplinary Studies. 2013; 1: 33-46.

39. Mokgolodi NC, Hu Yan SM, Ling-ling M, Yu-jun L. Ziziphus mucronata: An under-utilized traditional medicinal plant in Africa. Forestry Studies in China. 2011; 13: 163-172. 